\title{
Colonic Self-Expandable Metallic Stents for Patients with Colorectal Cancer Obstruction Induced Sepsis
}

\author{
Heng Fan, Jian-hua Zhu \\ Department of Intensive Care Unit, Ningbo First Hospital, Ningbo, China
}

Email address:

peterbenny@163.com (Jian-hua Zhu)

\section{To cite this article:}

Heng Fan, Jian-hua Zhu. Colonic Self-Expandable Metallic Stents for Patients with Colorectal Cancer Obstruction Induced Sepsis. Journal of Surgery. Vol. 5, No. 3, 2017, pp. 53-57. doi: 10.11648/j.js.20170503.14

Received: April 6, 2017; Accepted: April 27, 2017; Published: June 22, 2017

\begin{abstract}
To determine whether using colonic self-expandable metallic stents (SEMS) as a bridge to surgery for patients with colorectal cancer obstruction induced sepsis (CRCOIS) have better health outcomes compared with emergency surgery (ES). The patients with CRCOIS were enrolled from three university hospitals in China and retrospectively divided into SEMS group $(n=32)$ or ES group $(n=20)$. A total of 52 patients met the inclusion criteria. SEMS group had less the incidence of deep vein thrombosis $(\mathrm{P}<0.01)$ and number of organ dysfunction $(\mathrm{P}<0.01)$ than ES group. Kaplan-Meier curve showed no significant difference is found in term of 360-day overall survival between the two groups (log-rank test, $\mathrm{P}=0.12$ ). However, Multivariable analysis showed the pathological stages (OR: $1.26,95 \% \mathrm{CI}$ : 0.72-1.63, $\mathrm{P}<0.01)$ and number of organ failure (OR: $0.95,95 \% \mathrm{CI}$ : $0.71-1.42, \mathrm{P}<0.01)$ are independent risk factors for 360 -day overall survival. Therefore, SEMS placement as a bridge to surgery followed by selective surgery is a feasible and safe procedure for patients with CRCOIS, provides significant advantages of prognosis such as reducing the length of hospital stay, and decreasing the incidence of deep venous thrombosis and organ dysfunction.
\end{abstract}

Keywords: Self-expandable Metallic Stents, Colorectal Cancer Obstruction, Sepsis, Emergency Surgery

\section{Introduction}

Colorectal cancer (CRC) is one of the most common cancers worldwide [1] and the fourth most common cancer in China [2]. With the potential progression of CRC, $8-13 \%$ patients developed acute CRC obstruction, and 9-12\% of CRC obstruction patients induced sepsis [3-5]. Conventional emergency surgical treatments was always first choice in clinical practice for CRC obstruction patients. However, these patients may also have the imbalance of water and electrolytes as well as acid-base, and prone to develop multiple organ dysfunction. Moreover, most of these patients have poor overall physical health and longer time potential development, resulting in prolonged the hospital stay, increased short- and long-term mortality [3]. Thus, once these patients complicate with sepsis, the selection of surgical procedures is crucial for the outcomes.

Using a self-expandable metallic stent (SEMS) as a bridge to surgery (BTS) in CRC obstruction patients was introduced in the 1990s [6]. Thereafter, many studies have showed that SEMS placement followed by elective operation represents a relatively safe and simple alternative to conventional emergency surgery (ES) [7-10]. SEMS placement during pre-operation can prevent high-risk morbidity, decrease stoma creation and increase primary anastomosis [9]. However, for patients with colorectal cancer obstruction induced sepsis (CRCOIS), no related study results are reported.

To accurately evaluate whether using colorectal SEMS placement as a BTS for the patients with CRCOIS have better health outcomes compared with ES. In this study, we will investigate the outcomes of SEMS placement as a BTS for patients with CRCOIS in terms of demographical characteristics, treatments details, 360-day survival rate, and the incidence of deep venous thrombosis (DVT) and organ dysfunction.

\section{Method}

\subsection{Patients}

All patients admitted with acute CRCOIS in three university hospitals between January 2015 and November 
2016 were retrospectively collected in a database. Inclusion criteria: (1). aged $>18$ years; (2). Patients had clinical signs of acute colonic or rectal obstruction; (3). The gastrografin enema study or contrast-enhanced CT scan confirmed colon dilation and typical abnormalities; 5. Diagnosis of sepsis: 1) body temperature $>38.3^{\circ} \mathrm{C}$ or $<36^{\circ} \mathrm{C}, 2$ ) heart rate $>90 / \mathrm{min}$, 3) white blood cells $>12 \times 10^{9} / \mathrm{L}$ or $<4 \times 10^{9} / \mathrm{L}$, 4) systolic blood pressure $<90 \mathrm{mmHg}$ or mean blood pressure $<$ $70 \mathrm{mmHg}$. The patients with more than two above clinical symptoms will further test their blood, and if bacteria is detected in blood culture, the diagnosis of sepsis is confirmed [11]. Exclusion criteria: (1). Signs of peritonitis and perforation demanding urgent surgery; (2). the American Society of Anesthesiologists (ASA) classification of 4 or 5 [12]; (3). Obstruction caused by an adhesive ileus; (4). Patients can not complete self-report quality-of-life questionnaires; 5. Other reasons induced sepsis, such as intestinal perforation, diffuse peritonitis, and other parts of the infection. Our study was approved by the local ethics committee and the informed consents were not available because this was just a retrospective study and collected data were anonymously analyzed, the local ethics committee specifically waived the requirement for the informed consents.

\subsection{Procedures}

The SEMS was done by a combined fluoroscopic and endoscopic approach, performed by experienced colorectal surgeons and supervised by radiologists. If the lesion seemed to be benign by a standard colonoscope, the SEMS placement was not done. Balloon pre-dilatation before SEMS placement was not performed. Before SEMS placement was attempted, patients were given $120 \mathrm{ml}$ sodium phosphate to clean enemas. The SEMS used was the Amplatz super stiff wire. If SEMS placement failed (Guide wire or stent related perforations) or symptoms of colonic obstruction without remission within 3 days, patients were treated surgically. Patients received ES treatments were undergone as soon as possible after initial stabilization according to conventional standards. Surgical options included open or laparoscopic resection, segmental or subtotal colectomy. Primary anastomosis was at the discretion of the colorectal surgeons. During the study period, the surgical teams and postoperative protocols were not change.

\subsection{Data Collection}

The authors retrospectively reviewed the details of patient in the SEMS group and ES group, including age, gender, the pTNM stage, ASA scores, obstruction site, severity of obstruction, adjuvant postoperative chemotherapy, surgical procedure, postoperative mortality and adverse outcomes. Follow-up endpoint was defined as last radiological or clinical contact, or death. The primary outcome was 360-day mortality, and secondary outcomes were the adverse outcomes, including the incidence of DVT and organ dysfunction, anastomotic leakage, and electrolyte disturbance.

\subsection{Statistical Analysis}

SPSS software (version 18.0, IL, Chicago, USA) was used for calculation. For patient demographic and baseline clinical characteristics of both study groups, $\chi^{2}$ test or two-sided Fisher's exact test were used for categorical variables, MannWhitney U test was used if the continuous variables belonged to a no-normal distribution. Survival analysis was estimated using Kaplan-Meier survival curves and the significant differences of the findings were identified by a log-rank test. The clinical relevant variables for survival rate were analyzed by Multivariable logistic regression models, and statistical significance differences were used the Wald test. $\mathrm{P}<0.05$ was considered statistically significant.

\section{Results}

\subsection{Patient Characteristics and Baseline}

Between January 2013 and November 2016, the authors retrospectively identified 52 patients who met the included criteria from three university hospitals. Both of two groups (the SEMS group and ES group) were well balanced about clinical parameters, such as age, gender, and adjuvant chemotherapy status $(\mathrm{P}=0.15, \mathrm{P}=0.96$, and $\mathrm{P}=0.08$, respectively), etc. No differences were found between the two groups in terms of the obstruction site, pTMN stage of the CRC and severity of obstruction (Table 1). Blood culture results showed that Escherichia coli were main infectious bacterium of 34 cases $(65.4 \%)$, the second is Klebsiella pneumoniae of $12(22.6 \%)$, and $6(11.5 \%)$ cases were mixed bacterial infection (such as Escherichia coli, anaerobic bacteria and Klebsiella pneumoniae).

Table 1. Demographical and oncological characteristics of the study population.

\begin{tabular}{llll}
\hline & SEMS (n=32) & ES(n=20) & P value \\
\hline Mean age (year) & $59.5 \pm 9.8$ & $63.9 \pm 11.4$ & 0.15 \\
Male (n) & $19(59.4 \%)$ & $12(60 \%)$ & 0.96 \\
ASA score (n) & & & \\
I & $6(18.8 \%)$ & $3(15 \%)$ & 0.73 \\
II & $17(53.1 \%)$ & $11(55 \%)$ & 0.90 \\
III & $9(28 \%)$ & $7(35 \%)$ & 0.97 \\
pTNM stage (n)* & & & \\
I & $3(9.4 \%)$ & $1(5 \%)$ & 0.57 \\
II & $7(21.9 \%)$ & $4(20 \%)$ & 0.87 \\
III & $12(37.5 \%)$ & $8(40 \%)$ & 0.86 \\
IV & $10(31.3 \%)$ & $7(35 \%)$ & 0.78 \\
Obstructive site (n) & & & \\
Colon & $27(84.4 \%)$ & $18(90 \%)$ & 0.57 \\
Rectum & $5(15.6 \%)$ & $2(10 \%)$ & 0.57 \\
Severity of obstruction & & & \\
Unknown & $2(6.3 \%)$ & $1(5 \%)$ & 0.85 \\
Incomplete $\dagger$ & $25(78.1 \%)$ & $11(34.4 \%)$ & 0.08 \\
Complete & $5(15.6 \%)$ & $8(40 \%)$ & 0.05 \\
Time to surgery (days, & $21(0-62)$ & $0(0 \%)$ & \\
median) & & $1(5 \%)$ & 0.05 \\
Type of surgery (n) & $16(50 \%)$ & $1(5 \%)$ & 0.85 \\
Open & $13(40.6 \%)$ & & \\
Laparoscopic & $2(6.3 \%)$ & & \\
Converted & & & \\
Blood culture (n) & & & \\
\hline
\end{tabular}




\begin{tabular}{llll}
\hline & SEMS (n=32) & ES(n=20) & P value \\
\hline Escherichia coli & $21(65.6 \%)$ & $13(65 \%)$ & 0.96 \\
Klebsiella pneumoniae & $7(21.9 \%)$ & $5(25 \%)$ & 0.79 \\
Mixed bacteria & $4(12.5 \%)$ & $2(10 \%)$ & 0.78 \\
\hline
\end{tabular}

Values are presented as $n(\%) ; *$ The pathological stages of colorectal cancer; $\dagger$ Clinical signs of ileus but able to pass flatus; $\vdots$ Unable to pass flatus during the last $24 \mathrm{~h}$ before inclusion. SEMS: self-expanding metallic stents; ES: Emergency surgery; ASA: American Society for Anesthesiologists.

\subsection{The Features of SEMS and ES Group}

The overall SEMS insertion success rate was 75\% (24/32). During endoscopy, the guide-wire failure to pass through the obstruction site of tumor was in $15.6 \%$ of cases $(5 / 32)$. The median time was 21(0-62) days from SEMS placement to elective surgery. The 28-day mortality for postoperative patients was $18.8 \%$ (6/32), 180-days mortality was $34.4 \%$ $(11 / 32)$, and 360 -day mortality was $46.9 \%$ (15/32). DVT was found in $1(3.1 \%)$ patient, and organ dysfunction appeared in 4 $(12.5 \%)$ patients (Table 2$)$.

For ES group, the 28-day mortality for postoperative patients was $20 \%$ (4/20), 180-days mortality was $40 \%(8 / 20)$, and 360-day mortality was 55\% (11/20). The severe complications of ES patients were anastomosis leak (10\%), electrolyte disturbance (15\%) and wound infection (5\%). DVT was found in 7 (35\%) patients and organ dysfunction appeared in $10(50 \%)$ patients. The SEMS group has significantly less the incidence of DVT $(\mathrm{P}<0.01)$ and organs dysfunction $(\mathrm{P}<0.01)$ compare with ES group (Table 2$)$.

Table 2. Patients with complications.

\begin{tabular}{llll}
\hline Complications & SEMS (n=32) & $\begin{array}{l}\text { ES } \\
(\mathbf{n = 2 0})\end{array}$ & P value \\
\hline Abscess & $2(6.25 \%)$ & $1(5 \%)$ & 0.85 \\
Perforation & & & \\
Guide wire perforations & $2(6.25 \%)$ & $0(0 \%)$ & \\
Stent related perforations & $1(3.1 \%)$ & $0(0 \%)$ & \\
Anastomotic leakage & $1(3.1 \%)$ & $2(10 \%)$ & 0.33 \\
Electrolyte disturbance & $4(12.5 \%)$ & $3(15 \%)$ & 0.80 \\
Bleeding & $1(3.1 \%)$ & $1(5 \%)$ & 0.73 \\
Ileus again & $1(3.1 \%)$ & $0(0 \%)$ & \\
Constipation & $3(9.4 \%)$ & $2(10 \%)$ & 0.94 \\
Delirium & $3(9.4 \%)$ & $3(15 \%)$ & 0.54 \\
DVT & $1(3.1 \%)$ & $7(35 \%)$ & $<0.01$ \\
Number of admit to ICU & $17(53.1 \%)$ & $16(80 \%)$ & 0.06 \\
Organ dysfunction & $4(12.5 \%)$ & $10(50 \%)$ & $<0.01$ \\
Respiratory dysfunction & $2(6.25 \%)$ & $3(15 \%)$ & 0.31 \\
Renal dysfunction & $1(3.1 \%)$ & $3(15 \%)$ & 0.15 \\
Heart dysfunction & $1(3.1 \%)$ & $2(10 \%)$ & 0.33 \\
other & $1(3.1 \%)$ & $2(10 \%)$ & 0.33 \\
The length of hospital stay (days) & $13(8-21)$ & $15(11-19)$ & $<0.01$ \\
28-day mortality (n) & $6(18.8 \%)$ & $4(20 \%)$ & 0.91 \\
180-day mortality (n) & $9(28.1 \%)$ & $8(40 \%)$ & 0.38 \\
360-day mortality (n) & $15(46.9 \%)$ & $11(55 \%)$ & 0.57 \\
\hline
\end{tabular}

Values are presented as n (\%). SEMS: self-expanding metallic stents; ES: Emergency surgery; DVT: deep venous thrombosis; ICU: intensive care unit;

\subsection{Survival Rate}

The length of hospital stay was shorter in the SEMS group than ES group (Median: 13 versus 15 days, $\mathrm{P}<0.01$ ). During 360 days follow-up, $15(46.9 \%)$ patients in the SEMS group and $11(34.4 \%)$ patients in the ES group were died. Of the 26 patients who died, $12(46.2 \%)$ died of cancer, and $10(38.5 \%)$ died of organ failure, and 3 (11.5\%) died of unknown causes. The Kaplan-Meier curve showed no difference was found in the 360-day overall survival between the two groups (log-rank test, $\mathrm{P}=0.12$; Figure 1). However, Multivariable analysis showed the pTMN stage (OR: 1.26, 95\%CI: 0.72-1.63, P< 0.01 ) and number of organ failure (OR: 0.95 , 95\% $0.71-1.42, \mathrm{P}<0.01)$ were independent risk factors for 360 -day survival (Table 3).

Table 3. Multivariable analysis of known risk factors for 360-day mortality.

\begin{tabular}{llll}
\hline & Odds ratio & $\mathbf{9 5 \% ~ C I ~}$ & P value \\
\hline Age & 1.00 & $0.99-1.01$ & 0.97 \\
Gender & 0.71 & $0.47-1.01$ & 0.09 \\
pTMN stage & 1.26 & $0.72-1.63$ & $<0.01$ \\
ASA grade & 0.82 & $0.64-1.05$ & 0.11 \\
Obstructive site & 0.82 & $0.52-1.31$ & 0.41 \\
Severity of obstruction & 0.88 & $0.69-1.12$ & 0.30 \\
Number of organ failure & 0.95 & $0.71-1.42$ & $<0.01$ \\
DVT & 0.91 & $0.75-1.32$ & 0.11 \\
Chemotherapy & 1.42 & $0.95-2.11$ & 0.08 \\
SEMS & 1.03 & $0.71-1.51$ & 0.85 \\
ES & 0.87 & $0.63-1.21$ & 0.41 \\
\hline
\end{tabular}

ASA: American Society for Anesthesiologists; DVT: deep venous thrombosis; SEMS: self-expanding metallic stents; ES: Emergency surgery;

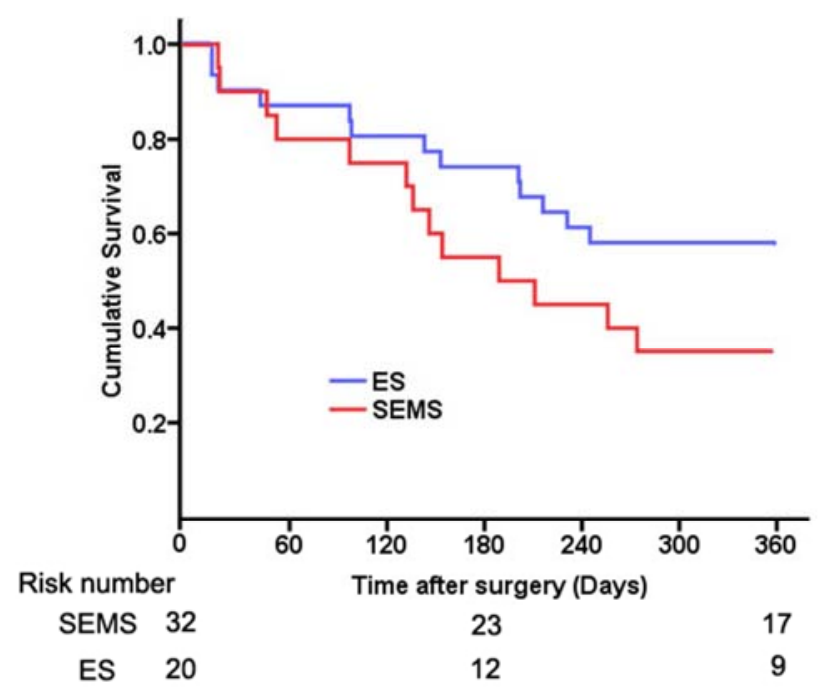

Figure 1. SEMS as a bridge to elective surgery versus ES for 360-day survival in patients with CRCOIS (log-rank test, $P=0.12$ ). (SEMS: self-expanding metallic stents; ES: Emergency surgery; CRCOIS: Colorectal cancer obstruction induced sepsis).

\section{Discussion}

Sepsis is one of the common and dreadful CRC obstruction complications [4], which do occur is always disastrous, especially in patients who need surgery to relieve the acute ileus. With the progression of disease, the septic patients prone to develop multiple organ dysfunction, and resulting in prolonged the hospital stay and increased hospital mortality [13]. Most of patients with CRC obstruction have poor overall physical health and longer time 
potential development [9]. Once these patients complicated with sepsis, the selection of surgical procedures is crucial for the short- and long-term outcomes. Our study is a comprehensive assessment of the patients with CRCOIS, and which in order to give general surgeon a clinical practical guide to improve the outcomes.

ES emerges many poor outcomes due to without sufficient preoperative preparation, such as the distended bowel and potential hazard of injury to the fragile bowel. However, SEMS placement was used as a BTS treatment may make up for these shortcomings. The SEMS was introduced in the 1990s [6], which can quick release of CRC related obstruction. Additionally, the SEMS placement will give general surgeon an opportunity to perform bowel preparation, optimization of comorbid disorders, and medical resuscitation [14, 15]. In a meta-analysis of large randomized controlled trials concluded that the SEMS placement as a BTS have many significant advantages, such as a shorter hospital stay, lower rate of stoma formation, and higher rate of primary anastomosis compared with ES [16]. In a recent study, Ho et al [17] also reported that SEMS as a BTS is a safe and feasible procedure, and have better short-term outcomes for acute malignant colorectal obstruction than ES.

Although preoperative SEMS placement has so many advantages, some SEMS related adverse outcomes also have been reported, such as re-obstruction, stent migration and perforation [18]. Morse et al [19] reported that SEMS insertion had 47.0-96.7 \% technical and 70.2-100\% clinical success, but with re-obstruction and perforation rates of $10-14 \%$ and $2-12 \%$, respectively [20]. One multicenter randomized trial from European also reported the SEMS placement had a high rate of perforation $(12.8 \%)$ and proposed no much clinical advantages compared with did ES [21, 22].

Until now, no study reported whether SEMS is useful for the patients with CRCOIS. In this study, we found that the SEMS placement followed by selective surgery has the advantages of lower postoperative morbidity and faster recovery compared with did ES. Comparison of the 360-day survival in the both subgroups found that SEMS approach could achieve better prognosis (no significant, Figure 1.), but less the incidence of DVT and organ dysfunction. Furthermore, laparoscopic surgery could be considered as an optimal type of surgery after SEMS placement. Therefore, colonic SEMS placement as a BTS is useful and may become a standardized treatment for patients with CRCOIS.

This study also indicated that number of organ dysfunction and pTMN stage were independent risk factors for 360-day survival (Table 3 ). It is crucial to relieve the acute obstructions as soon as possible and meanwhile protect organs function for these patients. ES may aggravate the multiple organ dysfunction and lead to worse clinical prognosis. Other treatments are also indispensable, such as strengthen anti-infection, adequate fluid resuscitation, balance water and electrolyte as well as acid-base, and the intensive care in the treatment may help to protect organs function and improve the prognosis.

This study has three weaknesses. Firstly, it is a retrospective observational study and the included number of patients is limited, heterogeneity may exist. Further study will need more high quality cases to full evaluate the advantages and disadvantages of SEMS placement for patients with CRCOIS. Secondly, blood bacterial culture will take more than two days, but ES often needs to make decision immediately after confirmed CRC obstruction, so full assessment should be taken for highly suspected sepsis before select a surgical approach. Thirdly, for other infection variables may affect the survival rate, such as procalcitonin, C-reaction protein, and leukocyte, were not included in this analysis.

\section{Conclusions}

SEMS placement as a BTS followed by selective surgery is a feasible and safe procedure and provides significant advantages of prognosis such as reducing the length of hospital stay, decreasing DVT rate and number of organ dysfunction, which is worthy of clinical application and popularization for patients with CRCOIS. Although SEMS may have these advantages for patients with CRCOIS, the number of this study is relatively few so that the heterogeneity may exist. A large randomized controlled study is needed to clarify the short- and long-term outcomes of SEMS in future studies.

\section{Acknowledgements}

Our study is supported by "The clinical scientific research fund of Zhejiang medical association (2015ZYC-A54)" and "Medical science and technology fund of Zhejiang province (2017KY134).

\section{References}

[1] Ferlay J, Autier P, Boniol M, Heanue M, Colombet M, Boyle P. Estimates of the cancer incidence and mortality in Europe in 2006. Ann Oncol. 2007, 18: 581-92.

[2] Xu M, Chen YM, Huang J, Fang YJ, Huang WQ, Yan B, Lu MS, Pan ZZ, Zhang CX. Flavonoid intake from vegetables and fruits is inversely associated with colorectal cancer risk: a case-control study in China. Br J Nutr. 2016, 116: 1275-87.

[3] Bertelsen CA, Neuenschwander AU, Jansen JE, Kirkegaard-Klitbo A, Tenma JR, Wilhelmsen M, Rasmussen LA, Jepsen LV, Kristensen B, Gögenur I; Copenhagen Complete Mesocolic Excision Study (COMES); Danish Colorectal Cancer Group (DCCG). Short-term outcomes after complete mesocolic excision compared with 'conventional' colonic cancer surgery. Br J Surg. 2016, 103: 581-9.

[4] Hsu CW, Wang JH, Kung YH, Chang MC. What is the predictor of surgical mortality in adult colorectal perforation? The clinical characteristics and results of a multivariate logistic regression analysis. Surg Today. 2016 Sep 20. [Epub ahead of print].

[5] Moghadamyeghaneh Z, Hanna MH, Carmichael JC, Mills SD, Pigazzi A, Stamos MJ. Preoperative Leukocytosis in Colorectal Cancer Patients. J Am Coll Surg. 2015, 221: 207-14. 
[6] Dohmoto M. New method: endoscopic implantation of rectal stent in palliative treatment of malignant stenosis. Endosc Dig. 1991, 3: 1507-12.

[7] Sagar J. Colorectal stents for the management of malignant colonic obstructions. Cochrane Database Syst Rev. 2011, 9: CD007378.

[8] Tan CJ, Dasari BVM, Gardiner K. Systematic review and meta-analysis of randomised clinical trials of self-expanding metallic stents as a bridge to surgery versus emergency surgery for malignant left-sided large bowel obstruction. Br J Surg. 2012, 99: 469-76.

[9] Kavanagh DO, Nolan B, Judge C, Hyland JM, Mulcahy HE, O'Connell PR, et al. A comparative study of short- and medium-term outcomes comparing emergent surgery and stenting as a bridge to surgery in patients with acute malignant colonic obstruction. Dis Colon Rectum. 2013, 56: 433-40.

[10] Mackay CD, Craig W, Hussey JK, Loudon MA. Self-expanding metallic stents for large bowel obstruction. Br J Surg. 2011, 98: 1625-9.

[11] Kochanek M, Shimabukuro-Vornhagen A, von Bergwelt-Baildon M, Böll B. Sepsis: new findings and developments. Update 2016. Dtsch Med Wochenschr. 2016, 141: $1455-8$.

[12] Greene NM. An A. S. A. (American Society of Anesthesiologists) Overseas Teaching Program. Anesthesiology. 1990, 72: 1-2.

[13] Mira JC, Gentile LF, Mathias BJ, Efron PA, Brakenridge SC, Mohr AM, Moore FA, Moldawer LL. Sepsis Pathophysiology, Chronic Critical Illness, and Persistent Inflammation -Immunosuppression and Catabolism Syndrome. Crit Care Med. 2016 Sep 14. [Epub ahead of print].

[14] Dastur JK, Forshaw MJ, Modarai B, Solkar MM, Raymond T, Parker MC. Comparison of short- and long-term outcomes following either insertion of self-expanding metallic stents or emergency surgery in malignant large bowel obstruction. Tech Coloproctol. 2008, 12: 51-5.
[15] Kim JS, Hur H, Min BS, Sohn SK, Cho CH, Kim NK. Oncologic outcomes of self-expanding metallic stent insertion as a bridge to surgery in the management of left-sided colon cancer obstruction: comparison with nonobstructing elective surgery. World J Surg. 2009, 33: 1281- 6 .

[16] Cirocchi R, Farinella E, Trastulli S, Desiderio J, Listorti C, Boselli C, Parisi A, Noya G, Sagar J. Safety and efficacy of endoscopic colonic stenting as a bridge to surgery in the management of intestinal obstruction due to left colon and rectal cancer: a systematic review and meta-analysis. Surg Oncol. 2013, 22: 14-21.

[17] Ho KM, Chan KM, Kwok SY, Lau PY. Colonic self-expanding metal stent (SEMS) as a bridge to surgery in left-sided malignant colonic obstruction: an 8-year review. Surg Endosc. 2016 Sep 8. [Epub ahead of print].

[18] Testini M, Margari A, Amoruso M, et al. The dehiscence of colorectal anastomoses: the risk factors. Ann Ital Chir. 2000, 71: 433-40.

[19] Morse BC, Simpson JP, Jones YR, Johnson BL, Knott BM, Kotrady JA. Determination of independent predictive factors for anastomotic leak: analysis of 682 intestinal anastomoses. Am J Surg. 2013, 206: 950-5.

[20] Saida Y, Enomoto T, Takabayashi K, Otsuji A, Nakamura Y, Nagao J, et al. Outcome of 141 cases of self-expandable metallic stent placements for malignant and benign colorectal strictures in a single center. Surg Endosc. 2011, 25: 1748-52.

[21] van Hooft JE, Fockens P, Marinelli AW, Bossuyt PM, Bemelman WA, Dutch Stent-In Study Group. Premature closure of the Dutch Stent-in I study. Lancet. 2006, 368: 15734.

[22] van Hooft JE, Bemelman WA, Oldenburg B, Marinelli AW, Lutke Holzik MF, Grubben MJ, Sprangers MA, Dijkgraaf MG, Fockens P; collaborative Dutch Stent-In study group. Colonic stenting versus emergency surgery for acute left-sided malignant colonic obstruction: a multicentre randomised trial. Lancet Oncol. 2011, 12: 344-52. 